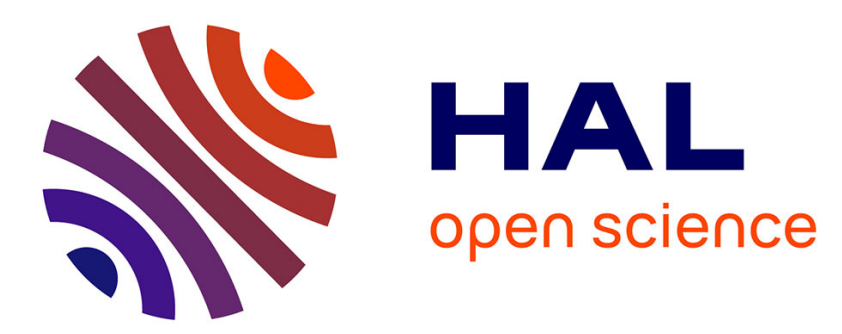

\title{
A compact model of precessional spin-transfer switching for MTJ with a perpendicular polarizer
}

\author{
Abdelilah Mejdoubi, Guillaune Prenat, Bernard Dieny
}

\section{To cite this version:}

Abdelilah Mejdoubi, Guillaune Prenat, Bernard Dieny. A compact model of precessional spin-transfer switching for MTJ with a perpendicular polarizer. 28th International Conference on Microelectronics (MIEL 2012), IEEE, May 2012, Nis, Serbia. 10.1109/MIEL.2012.6222840 . hal-00922298

\section{HAL Id: hal-00922298 \\ https://hal.science/hal-00922298}

Submitted on 30 Dec 2013

HAL is a multi-disciplinary open access archive for the deposit and dissemination of scientific research documents, whether they are published or not. The documents may come from teaching and research institutions in France or abroad, or from public or private research centers.
L'archive ouverte pluridisciplinaire HAL, est destinée au dépôt et à la diffusion de documents scientifiques de niveau recherche, publiés ou non, émanant des établissements d'enseignement et de recherche français ou étrangers, des laboratoires publics ou privés. 


\title{
A compact model of precessional spin-transfer switching for MTJ with a perpendicular polarizer
}

\author{
A. Mejdoubi, G. Prenat, and B. Dieny
}

\begin{abstract}
Magnetic Tunnel Junction (MTJ) devices are CMOS compatible with high stability, high reliability and non-volatility. A macro-model of MTJ with precessional switching is presented in this paper. This model is based on Spin-Transfer Torque (STT) writing approach. The current-induced magnetic switching and excitations was studied in structures comprising a perpendicularly magnetized polarizing layer $(P L)$, an in-plane magnetized free layer $(F L)$, and an in-plane magnetized analyzing layer $(A L)$.
\end{abstract}

\section{INTRODUCTION}

Magnetic Tunnel Junctions (MTJ) in CMOS [1] have become the cornerstone of spin electronics device such as magnetic random access memory (MRAM). As predicted by Slonczewski [2] and Berger [3], a spin polarized current flowing through a magnetoresistive nanopillar exerts a torque on the local magnetization. This phenomenon, called Spin-Transfer Torque (STT), is currently considered as a very promising write scheme for MRAM devices, offering low power consumption and good scalability as a function of the lateral cell size. Such MRAM devices usually comprise a free layer $(F L)$ and polarizing layer $(P L)$ structure, where FL and PL are the free and pinned layer, respectively, the latter playing the role of a spin polarizer. In order to enhance the writing speed of MRAM devices, the duration of the $F L$ switching should be as short as possible. A strategy to achieve sub-ns switching times, is to take advantage of the precessional motion of the magnetization around the effective field direction before it aligns itself with this direction.

In this work, we investigate a scalable MTJ concept that uses two polarizers: one perpendicularly $(P L)$ and one longitudinally $(A L)$ magnetized as sketched in Fig. 1. Up to now, the efforts to reduce the energy required to write by STT have been mainly focused on reducing the STT write critical current density [4]. This decrease in write current actually reduces the current value that the selection transistor has to sink/drive. Correlatively, smaller transistor sizes can be achieved. However, another possibility to reduce the total energy used to write one bit is to reduce the time that current/power needs to be applied to switch the magnetic configuration of the storage element. If the current density remains

A. Mejdoubi, G. Prenat, and B. Dieny are with the Laboratory of Spintronique et Technologie des Composants, Spintec/CEA/CNRS/UJF, 17, rue des Martyrs, 38054 Grenoble, France, E-mail: abdelilah.mejdoubi@cea.fr constant, reducing the pulse time from $20 \mathrm{~ns}$ to $0.2 \mathrm{~ns}$ reduces the total energy by a factor 100 . To achieve sub-ns switching times, precessional STT switching can be used [5]. This approach is useful for the design of hybrid MTJ/CMOS circuits dedicated to logic applications, which require very high speed.

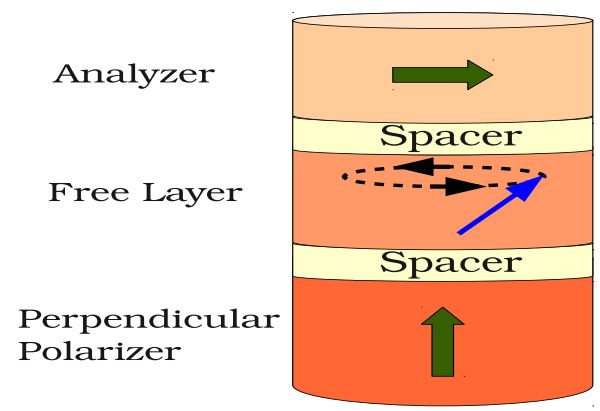

Fig. 1. Schematics of the perpendicular-polarize STT-MRAM cell, comprising a perpendicular polarizer, a first nonmagnetic spacer layer made of $\mathrm{Cu}$ or low R.A tunnel barrier, a planar free layer, a tunnel barrier and a third planar analyzing layer.

\section{Physical MOdel integration}

Magnetic Tunnel Junctions are used as the basic memory elements in MRAM cells and magnetic logic devices. They are composed essentially of three layers: a pinned layer and a storage layer, which are made of ferromagnetic materials (e.g. $\mathrm{CoFeB}$ ) separated by an insulating layer, such as $\mathrm{MgO}$. The magnetization in the pinned layer is fixed, whereas it can be switched in the storage layer. There are two stable configurations of the MTJ: parallel and anti-parallel alignement of the magnetizations in the storage and reference layers. The transitions between parallel and anti-parallel states present an hysteretic behavior (Fig. 2). Depending on the relative orientation of the magnetizations, the stack resistance varies: the resistance in the antiparallel state $\left(R_{A P}\right)$ is larger than the resistance in the parallel state $\left(R_{P}\right)$. The Tunnel Magneto Resistance is defined as the relative resistance variation between these two states: $T M R=\frac{R_{A P}-R_{P}}{R_{P}}$. The digital information is coded by the orientation of the magnetization of the storage layer. Reading information consists in measuring the MTJ resistance. This can be carried out, for example, by biasing the junction to a given voltage and reading the resulting current (Fig. 3). Writing a MTJ 


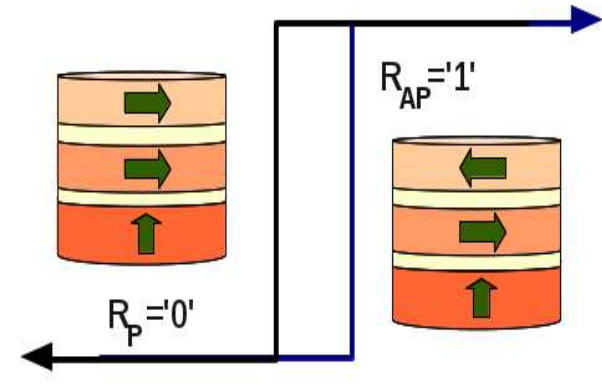

Fig. 2. Schematic representation of the resistance variation in a MTJ with additional perpendicular polarizer. The transitions between different resistance values are hysteretic.

cell can be performed by different methods, corresponding to different MRAM generations. In STT-MRAM, at write, the selection transistor is on and a spin-polarized current passes through the junction. If the current density is large enough, the soft layer magnetization can be reversed due to the resulting STT effect.

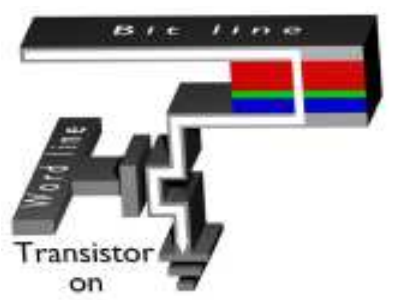

Fig. 3. Reading a MTJ (STT writing approach).

The magnetization dynamics of the soft layer is described by the Landau-Lifshitz-Gilbert (Eq. 1.):

$$
\begin{aligned}
\frac{\partial \vec{m}}{\partial t}= & -\frac{\gamma_{0}}{1+\alpha^{2}} \vec{m} \times \vec{H}_{e f f}-\alpha \frac{\gamma_{0}}{1+\alpha^{2}} \vec{m} \times\left(\vec{m} \times \vec{H}_{e f f}\right) \\
& -a_{J L O N G} V \vec{m} \times\left(\vec{m} \times \vec{p}_{L O N G}\right) \\
& +a_{J P E R P} V \vec{m} \times\left(\vec{m} \times \vec{p}_{P E R P}\right)
\end{aligned}
$$

where $\vec{m}$ represent the relative magnetization vector of the free layer, with $\left|m^{2}\right|=1, \vec{p}_{L O N G}$ and $\vec{p}_{P E R P}$ are the unit vectors along the polarizations of the plane (analyzer) and hard (polarizer) layers, respectively. $\vec{H}_{\text {eff }}$ is the effective field which consists of the magnetocrystalline anisotropy field, the demagnetizing field and the external field, $\alpha$ is the Gilbert damping parameter, $\gamma_{0}$ is the magnitude of the gyromagnetic ratio, $V$ is the bias voltage and $a_{J}=\frac{\bar{h}}{2 e} \frac{g(\theta)}{\mu_{0} M_{s} t} J, e$ is the electronic charge, $M_{s}$ is the saturation magnetization of the free layer, $t$ is the layer thickness and $g(\theta)$ is the spin-torque efficiency factor from Slonczewski model [2]; it is equal to the polarization $\vec{p}$ of the polarizing layer for a tunnel junction and, in the case of metallic spacer, it depends on the angle $\theta$ between the magnetization of the free layer and the magnetization of the polarizer or analyzer.

In the initial state, the parallel state, we find that the STT coming from LONG vanishes because the crossproduct $m \times m_{L O N G}$ vanishes, whereas the crossproduct $m \times m_{P E R P}$ is maximal.

The critical current density of LONG for magnetic excitations is derived from an instability condition of the easy plane component of magnetization [6]:

$$
J_{C}^{L O N G}=\alpha \frac{2 e}{\bar{h}} \frac{M_{s} \cdot t}{g(\theta=0)}\left(H_{K}+2 \pi M_{s}\right)
$$

where $H_{K}$ is the effective in-plane anisotropy field originating from the crystalline and the shape anisotropy.

In contrast, the critical current density for in-plane magnetic switching in PERP based structures can be derived from an instability condition of the out-of-plane component of magnetization $\left(m_{z}\right)$. This stems from the current induced switching mechanism with PERP: the flow of out-of-plane spin-polarized incoming electrons tends to drag the magnetization of the soft layer slightly out-of-plane. This yields the onset of a large perpendicular demagnetizing field around which the magnetization of the soft layer precesses. Therefore, in the case of PERP, the critical currents are given by [6]:

$$
J_{C}^{P E R P}=\frac{2 e}{\bar{h}} \frac{M_{s} \cdot t}{g\left(\theta=\frac{\pi}{2}\right)} \frac{H_{K}}{2}
$$

We introduce the factor to describe the relative spin transfer efficiency of the two polarizing layers $r=$ $\frac{a_{J L O N G}}{a_{J P E R P}}$. In section IV, we present the results corresponding to $r=10$ for different polarities of current to characterize the magnetic dynamics in the studied structures combining a perpendicular polarizer with inplane magnetized MTJ.

The dependence of the resistance on temperature is usually explained by elastic and inelastic tunneling [7]. In the present model, the temperature dependence of conductance for parallel and antiparallel states is given by:

$$
G_{P}=G_{T}\left[1+P_{1} P_{2}\right]+s T^{1.33}
$$

and

$$
G_{A P}=G_{T}\left[1-P_{1} P_{2}\right]+s T^{1.33}
$$

where $G_{T}=G_{0} \frac{C T}{\sin C T}, P_{1}$ and $P_{2}$ are the effective tunneling electron spin polarizations of the two ferromagnetic electrodes, and $C=1.387 \times 10^{4} t / \sqrt{\phi}$, with the barrier thickness $(t)$ in angstroms and the barrier height $(\phi)$ in electron-volts. $G_{0}$ is the conductance of the MTJ at zero temperature. In Eq. 4, the first part represents the elastic tunneling conductance, and the second part represents the inelastic one. 
It is assumed that the tunneling spin polarization follows the same temperature dependence as surface magnetization, Bloch $T^{3 / 2}$ law. This means we can write the spin polarization at temperature $T$ as:

$$
P(T)=P_{0}\left(1-\beta T^{3 / 2}\right)
$$

The parameter $P_{0}$ is the polarization of the electrode at zero temperature. The constant $\beta$ is a materialdependent constant. It describes the exchange coupling in the direction perpendicular to the surface. The parameter $\beta$ is generally larger for the surface than the bulk due to surface exchange softening.

\section{SPICE MODELLING}

Computer-aided-design (CAD) tools play a significant role in modern circuit design. With device models, we can simulate and verify the functionality of circuits to avoid failures before fabrication. However, the modeling of MTJs for circuit simulation purposes is still in its initial stage. In order to estimate the validity of architectures based on MTJ, performant compact models were developed in compiled $\mathrm{C}$ language, compatible with CADENCE SPECTRE simulator. This choise was motivated by a collaboration with CADENCE, but the models could be adapted to others simulations. This models, describe the electrical behaviour of the junctions $[8]$.

Any conventional circuit elements, such as resistor, capacitor or MOS transistor, can be considered as a "black box" with certain nodes in the circuit description. A circuit is then described as a collection of all these black boxes interconnected with each other. Voltages at any nodes in the circuit are calculated by using the nodal analysis technique based on Kirchhoffs current law (KCL). In SPICE, a circuit element with $n$ nodes can be represented by a $n \times n$ resistance matrix or capacitance matrix [8]. For example, a resistor has two nodes and it can be described by the matrix $\left[\begin{array}{cc}\frac{1}{R} & -\frac{1}{R} \\ -\frac{1}{R} & \frac{1}{R}\end{array}\right]$, where the matrix entries are derived from the resistors node voltages and branch currents. Regarding the MTJ, due to its unique characteristics, it cannot be modelled as other conventional circuit elements. The current that can pass through the junction is closely related to its magnetization state and working temperature. Its physical equations are not compatible with any existing equivalent RC components in SPICE. Therefore, much complex work has been carried out to reformulate its physical equations. The MTJ matrix entries are very complex mathematical functions derived from those reformulated equations. The presented MTJ model includes nine nodes, of which four are external nodes for real circuit connections and especially five internal virtual nodes for constructing virtual equivalent circuits to describe its electrical, magnetic and thermal
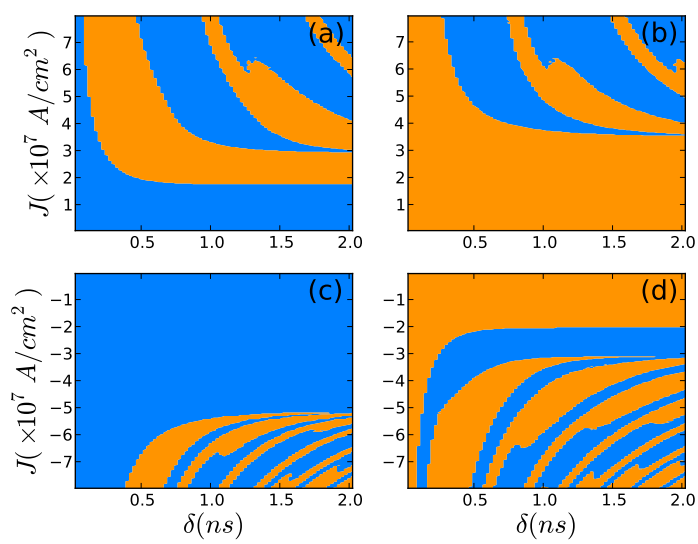

Fig. 4. Macrospin simulation of precessional switching $(r=10)$ at $0 \mathrm{k}$. Probability $P_{s}$ it plotted as a fonction of current pulse $\delta$ and density amplitude $J$. The blue and orange colors correspond respectively to parallel and anti-parallel case. (left panel) the initial state is parallel. (right panel) the initial state is a anti-parallel.

behaviours. This yield a large $9 \times 9$ matrix to be used to represent the MTJ in SPICE. In order to follow the usual SPICE representation for the virtual circuits, the three components of the magnetization $\left(m_{x}, m_{y}\right.$ and $m_{z}$ ) and the temperature are treated as virtual voltages in the simulation. The presented SPICE-like MTJ model is compatible with the SPECTRE simulator.

\section{RESUlts AND DISCUSSION}

In this section, we present results of macrospin simulations of precessional STT switching at 0K in the studied structures. Fig. 4. shows the switching probability $P_{s}$ as a function of current duration $\delta$ and current density $J$, assuming a rectangular pulse profile. The four following cases were considered: (1) $\mathrm{P}$ to AP with $J>0$, (2) AP to $\mathrm{P}$ with $J>0$, (3) $\mathrm{P}$ to AP with $J<0$ and (4) AP to $\mathrm{P}$ with $J<0$. The switching diagram consists of alternating blue and orange stripes corresponding to either "no switching" or "switching". Since no stochastic effects are present at $0 \mathrm{~K}, P_{s}$ is either 0 or 1 . The general shape on these stripes follows a hyperbolic variation. When electrons flow from $F L$ to $P L$ (Fig. 4(c)), the stripes are narrower than for the opposite current direction (Fig. 4(a)). The same remark holds for Fig. 4 (b) and (d), when electrons flow from $F L$ to $P L$ (Fig. $4(\mathrm{~d})$ ). The stripes are narrower than for the opposite current direction (Fig. 4(b)). If we now consider the difference between the two cases: (1) P to AP (Fig. 4 (a) and (c)) and (2) AP to P (Fig. 4 (b) and (d)), clearly we show that an even stronger asymmetry occurs as a function of current direction. A complex dynamic interplay takes place between the STT influence from $P L$ and $A L$, which changes the width of each individual stripe as well as their frequency. The corre- 


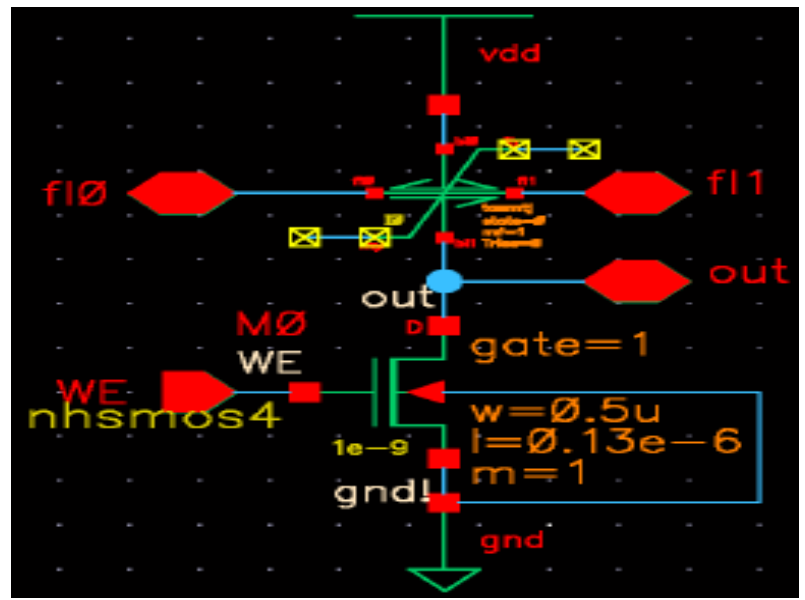

Fig. 5. Schematic window of a circuit drawn in Cadence design suite. In this figure, we can see the symbol representing the MTJ, the symbol of a transistor as well as a current and a voltage generator.

sponding precession frequency $f$ is proportional to the current density $J$, Eq. 7 [9]. This complex dynamics gives rise to reentrant switching probability, as observed in Fig. 4.

$$
f=\frac{\gamma}{2 \pi} \frac{\bar{h}}{2 e} \frac{g(\eta)}{M_{s} t} \frac{J}{\alpha}
$$

Fig. 5. present the schematic window describing a STT memory cell in Cadence: it consists of a MTJ connected with a selection transistor. The simulation stimuli are applied by a voltage generator to polarize the transistor. In this figure, two inputs BL0 and BL1 represent the two electrodes of the junction which is modelled by its tunnelling conductance and capacitance. The two nodes FL0 and FL1, are not used in this paper.

Fig. 6. shows simulation results of this cell. The switching behaviour can be detected by plotting the corresponding current through the MTJ and the internal node $m_{y}$. Since the initial conductance is high ( $\mathrm{P}$ state), the resulting current before switching is lower. In contrast, a lower current flows through the junction after switching because the device conductance changes to low (AP state).

\section{Conclusion}

We have developped an electrical macro-model describing the precessional STT switching in in-plane magnetized MTJ with additional perpendicular polarizer. The model allows to simulate hybrid STT based MTJ and CMOS architectures. These architectures have significant advantages in terms of power consumption reduction, performance improvement and new functionalities. It has been shown that a perpendicular polarizer can induce precessional reversal on a

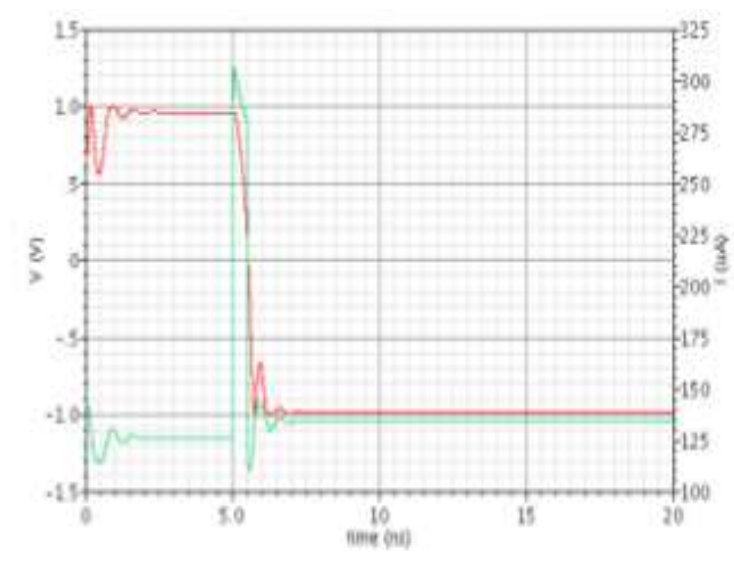

Fig. 6. Simulation results for STT cell: MTJ is switched between the $\mathrm{P}$ and AP (red color). Resulting tunnelling current through the MTJ (green color).

subnanosecond time scale, but that the coherence of the precessional switching is affected by the STT influence of the analyzer.

\section{ACKNOWLEDGMENT}

This work was supported by the European Commission through the ERC Adv.grant HYMAGINE.

\section{REFERENCES}

[1] WS, Zhao, E. Belhaire, C. Chappert, and P. Mazoyer, Spin transfer torque (STT)-MRAM-based runtime reconfiguration FPGA circuit, ACM Trans Embedded Computing, 9: article 14 (2009)

[2] J. C. Slonczewski, Current-driven excitation of magnetic multilayers, J. Magn. Magn. Mater. 159, L1 (1996)

[3] L. Berger, Emission of spin waves by a magnetic multilayer traversed by a current, Phys. Rev. B 54, 9353 (1996)

[4] D. C. Ralph, and M. D. Stiles, Spin transfer torques, J. Magn. Magn. Mater. 320, (2008)

[5] O. Redon, B. Dieny, and B. Rodmacq, Magnetic spin polarisation and magnetisation rotation device with memory and writing process, using such a device, patent US6532164B2 (2000)

[6] K. J. Lee, O, Redon, and B. Dieny, Analytical investigation of spin-transfert dynamics using a perpendicular-to-plane polarizer, APL. 86, 022505 (2005)

[7] C. H. Shang, J. Nowak, R. Jansen, and J. S. Moodera, Temperature dependence of magnetoresistance and surface magnetization in ferromagnetic tunnel junctions, Phys. Rev. B 58, R2917 (1998)

[8] W. Guo, G. Prenat, V. Javerliac, M. El Baraji, N. de Mestier, C. Baraduc, and B Dieny, SPICE modelling of magnetic tunnel junctions written by spin-transfer torque, J. Phys. D: Appl. Phys. 43215001 (2010)

[9] U. Ebels, D. Houssameddine, I. Firastrau, D. Gusakova, C. Thirion, B. Dieny, and L. D. Buda-Prejbeanu1, Macrospin description of the perpendicular polarizer-planar free-layer spin-torque oscillator, Phys. Rev. B 78, 024436 (2008) 